\title{
Erratum to: Operator renewal theory and mixing rates for dynamical systems with infinite measure
}

\author{
Ian Melbourne $^{1}$ - Dalia Terhesiu ${ }^{2}$
}

Published online: 12 August 2015

(C) Springer-Verlag Berlin Heidelberg 2015

\section{Erratum to: Invent Math (2012) 189:61-110 DOI 10.1007/s00222-011-0361-4}

We correct the statement and proof of Theorem 10.4 in our paper "Operator renewal theory and mixing rates for dynamical systems with infinite measure" [Inventiones Mathematicae 189 (2012) 61-110]. The main results in the paper are unaffected.

In the original article, we developed a theory of operator renewal sequences in the context of infinite ergodic theory. For large classes of dynamical systems preserving an infinite measure, we determine the asymptotic behaviour of iterates $L^{n}$ of the transfer operator, yielding results on mixing and rates of mixing.

The main results, presented in Section 2 of the original article, focus on the restricted transfer operator $1_{Y} L^{n} 1_{Y}$ where $Y$ is a suitable finite measure subset of the full infinite measure system, and accordingly yield mixing results for observables supported on $Y$. Extensions to more general observables were described in specific cases in Section 1 based on results described in Sections 10 and 11.

The online version of the original article can be found under doi:10.1007/s00222-011-0361-4.

$凶$ Ian Melbourne

i.melbourne@warwick.ac.uk

1 Mathematics Institute, University of Warwick, Coventry CV4 7AL, UK

2 Faculty of Mathematics, University of Vienna, 1090 Vienna, Austria 
Kautzsch et al. [1] have pointed out that there is an error in Theorem 10.4 of the original article. Here, we give a corrected statement and proof of this result, and indicate the implications of the changes. The body of the paper, namely the statement of the main results in Section 2 and their proof in Sections 3-9, is unaffected.

Throughout, the numbering and notation used here correspond to those in the original article. We recall that $f: X \rightarrow X$ is an ergodic conservative measure-preserving transformation of an infinite measure space $(X, \mu)$. We fix a subset $Y \subset X$ with $\mu(Y)=1$ and define the first return map $\varphi: Y \rightarrow \mathbb{Z}^{+}$, $\varphi(y)=\inf \left\{n \geq 1: f^{n} y \in Y\right\}$ together with the first return map $F=f^{\varphi}:$ $Y \rightarrow Y$. The key assumptions are that $\mu(y \in Y: \varphi(y)>n)=\ell(n) n^{-\beta}$ where $\beta \in(0,1]$ and $\ell$ is slowly varying, and that the first return map $F: Y \rightarrow Y$ is uniformly expanding in an appropriate sense. Let $L: L^{1}(X) \rightarrow L^{1}(X)$ denote the transfer operator for $f: X \rightarrow X$. Our main result, Theorem 2.1, yields results on the asymptotics of $1_{Y} L^{n} 1_{Y}$ acting on a suitable Banach space $\mathcal{B} \subset L^{\infty}(Y)$.

We begin by stating and proving the corrected version of Theorem 10.4. Define $X_{k}=f^{-k} Y \backslash \bigcup_{j=0}^{k-1} f^{-j} Y$ and $v_{k}=1_{X_{k}} v$. Noting that $L^{k} v_{k}$ is supported on $Y$ for all $k$, we write $v \in \mathcal{B}(X)$ if $v \in L^{1}(X)$ and $L^{k} v_{k} \in \mathcal{B}$ for all $k \geq 0$. Define $m(n)=\left\{\begin{array}{ll}\ell(n), & \beta<1 \\ \sum_{j=1}^{n} \ell(j) j^{-1}, & \beta=1\end{array}\right.$.

Theorem 10.4 Let $\beta \in\left(\frac{1}{2}, 1\right]$. Suppose that $v \in \mathcal{B}(X)$ and moreover that $\sum_{n=0}^{\infty}\left\|L^{n} v_{n}\right\|<\infty$ and either $(i)\left\|L^{n} v_{n}\right\|=o\left(n^{-1}\right)$ or $(i i) \sum_{k=n}^{\infty}\left\|L^{k} v_{k}\right\|=$ $o\left(\left(m(n) n^{1-\beta}\right)^{-1}\right)$. Then $\lim _{n \rightarrow \infty} m(n) n^{1-\beta} L^{n} v=d_{\beta} \int_{X} v d \mu$ uniformly on $Y$ and pointwise on $X$.

Proof Let $w(n)=d_{\beta}^{-1} m(n) n^{1-\beta}$. First we show that

$$
w(n) \sum_{n / 2 \leq j \leq n} w(n-j)^{-1}\left\|L^{j} v_{j}\right\| \rightarrow 0 .
$$

In case (i), $\left\|L^{n} v_{n}\right\|=o\left(n^{-1}\right)$, it follows from Karamata that

$$
\begin{aligned}
& w(n) \sum_{n / 2 \leq j \leq n} w(n-j)^{-1}\left\|L^{j} v_{j}\right\| \ll n^{-1} w(n) \sum_{n / 2 \leq j \leq n} w(n-j)^{-1}\left(j\left\|L^{j} v_{j}\right\|\right) \\
& \quad \leq n^{-1} w(n)\left(\sum_{n / 2 \leq j \leq n} w(n-j)^{-1}\right) \max _{j \geq n / 2} j\left\|L^{j} v_{j}\right\| \ll \max _{j \geq n / 2} j\left\|L^{j} v_{j}\right\| \rightarrow 0 .
\end{aligned}
$$


In case (ii),

$$
\begin{aligned}
& w(n) \sum_{n / 2 \leq j \leq n} w(n-j)^{-1}\left\|L^{j} v_{j}\right\| \ll w(n) \sum_{n / 2 \leq j \leq n}\left\|L^{j} v_{j}\right\| \\
& \quad \ll w(n / 2) \sum_{j \geq n / 2}\left\|L^{j} v_{j}\right\| \rightarrow 0 .
\end{aligned}
$$

Hence $(*)$ is verified in both cases.

Next we prove uniform convergence on $Y$. Let $c_{j, n}=\frac{w(n)}{w(n-j)}-1$. By Theorem 2.1, $T_{n} v=w(n)^{-1} \int_{Y} v d \mu+S_{n} v$ where $S_{n}=o\left(w(n)^{-1}\right)$. Hence on $Y$,

$$
\begin{aligned}
w(n) L^{n} v-\int v= & w(n) \sum_{j=0}^{n} T_{n-j} L^{j} v_{j}-\sum_{j=0}^{\infty} \int v_{j} \\
= & w(n) \sum_{j=0}^{n} w(n-j)^{-1} \int L^{j} v_{j}-\sum_{j=0}^{n} \int L^{j} v_{j} \\
& +w(n) \sum_{j=0}^{n} S_{n-j} L^{j} v_{j}-\sum_{j>n} \int v_{j} \\
= & \sum_{j=0}^{n} c_{j, n} \int L^{j} v_{j}+w(n) \sum_{j=0}^{n} S_{n-j} L^{j} v_{j}-\sum_{j>n} \int v_{j},
\end{aligned}
$$

and so

$$
\left|w(n) L^{n} v-\int v\right| \leq \sum_{j=0}^{n}\left|c _ { j , n } \left\|\int L^{j} v_{j}\left|+w(n) \sum_{j=0}^{n}\left\|S_{n-j}\right\|\left\|L^{j} v_{j}\right\|+\right| \sum_{j>n} \int v_{j} \mid .\right.\right.
$$

It is immediate that the third term converges to zero. Write $\left\|S_{n}\right\|=$ $w(n)^{-1} a_{n}$ where $a_{n}=o(1)$. Then the second term satisfies

$$
\begin{aligned}
& w(n) \sum_{j=0}^{n}\left\|S_{n-j}\right\|\left\|L^{j} v_{j}\right\| \\
& =w(n) \sum_{0 \leq j<n / 2} w(n-j)^{-1} a_{n-j}\left\|L^{j} v_{j}\right\| \\
& \quad+w(n) \sum_{n / 2 \leq j \leq n} w(n-j)^{-1} a_{n-j}\left\|L^{j} v_{j}\right\|
\end{aligned}
$$




$$
\begin{aligned}
& \ll \sum_{0 \leq j<n / 2} a_{n-j}\left\|L^{j} v_{j}\right\|+w(n) \sum_{n / 2 \leq j \leq n} w(n-j)^{-1}\left\|L^{j} v_{j}\right\| \\
& \leq\left(\sum_{k=0}^{\infty}\left\|L^{k} v_{k}\right\|\right) \max _{j \geq n / 2} a_{j}+w(n) \sum_{n / 2 \leq j \leq n} w(n-j)^{-1}\left\|L^{j} v_{j}\right\| \rightarrow 0
\end{aligned}
$$

by (*), summability of $\left\|L^{k} v_{k}\right\|$ and the definition of $a_{n}$.

The first term satisfies

$$
\begin{aligned}
\sum_{j=0}^{n}\left|c_{j, n}\right|\left|\int L^{j} v_{j}\right| & \leq \sum_{0 \leq j<n / 2}\left|c_{j, n}\right||| L^{j} v_{j}\left\|+\sum_{n / 2 \leq j \leq n}\left|c_{j, n}\right|\right\| L^{j} v_{j} \| \\
& \ll \sum_{0 \leq j<n / 2}\left|c_{j, n}\right|\left\|L^{j} v_{j}\right\|+w(n) \sum_{n / 2 \leq j \leq n} w(n-j)^{-1}\left\|L^{j} v_{j}\right\| .
\end{aligned}
$$

Again $w(n) \sum_{n / 2 \leq j \leq n} w(n-j)^{-1}\left\|L^{j} v_{j}\right\| \rightarrow 0$ by (*). Since $\lim _{n \rightarrow \infty} c_{j, n}=$ 0 for each fixed $j$ and $\max _{j, n: 0 \leq j<n / 2}\left|c_{j, n}\right|<\infty$, it follows from summability of $L^{n} v_{n}$ that $\sum_{0 \leq j<n / 2}\left|c_{j, n}\right|\left\|L^{j} v_{j}\right\| \rightarrow 0$. This completes the proof of uniform convergence on $Y$.

The proof of pointwise convergence on $X$ now goes through unchanged from the original article.

Theorem 10.4 is used in Section 11 first in the context of Thaler maps, and then for AFN maps, and proofs are given for various results mentioned in the Introduction of the original article. In particular, Theorem 11.8 and Remarks 11.9 and 11.11 need to be modified accordingly. We refer to [2] for an updated version which includes all the required modifications. For the Introduction, it suffices to strengthen slightly the hypotheses on the observable $v$ in Theorem 1.1 and Remark 1.2(iv). The remaining results in the Introduction, namely Theorem 1.3 and the discussion of second order asymptotics and rates of mixing, are unaffected. Finally the statement and proof of Theorems 10.6 and 11.14 are modified in [2]; the latter is still sufficient for Remark 1.2(iii). In particular, the statement in Remark 1.2(iii) that a wider class of observables is covered by our methods in relation to previous examples remains intact.

\section{References}

1. Kautsch, J., Kesseböhmer, M., Samuel, T., Stratman, B.O.: On the asymptotics of the $\alpha$-Farey transfer operator. Nonlinearity 28, 143-166 (2015)

2. Melbourne, I., Terhesiu, D.: Corrected version of the original article. arXiv:1008.4113v3 (2015) 